\title{
Feasibility of lung clearance index in a clinical setting in pre-school children
}

\author{
Barrett Downing, Samantha Irving, Yvonne Bingham, Louise Fleming, \\ Andrew Bush and Sejal Saglani
}

\section{Affiliation:}

NHLI, Imperial College London, and Royal Brompton and Harefield NHS Foundation Trust, London, UK.

\section{Correspondence:}

Sejal Saglani, Imperial College London, 368 Sir Alexander Fleming Building, London SW7 2AZ, UK.

E-mail: s.saglani@imperial.ac.uk

ABSTRACT Lung function testing in pre-school children in the clinical setting is challenging. Most cannot perform spirometry and many infant lung function tests require sedation. Lung clearance index (LCI) derived from the multiple-breath washout (MBW) test has been shown to be sensitive to early disease changes but may be time consuming and so a shortened test (LCI0.5) may be more feasible in young children. We sought to establish feasibility of MBW in unsedated pre-school children in a clinic setting and hypothesised use of LCI0.5 would increase success rates.

116 pre-school children ( 28 healthy controls and 88 with respiratory disease), median age 4.0 years (range 2-6 years), underwent MBW tests unsedated in a clinic setting, using sulfur hexafluoride as a tracer gas and an adapted photoacoustic gas analyser.

$81(70 \%)$ out of 116 children completed LCI and $72 \%$ completed LCI0.5 measurement. Test success increased significantly in patients over 3 years $(0 \%$ at $<2.5$ years, $33 \%$ at $2.5-3$ years and $70 \%$ at $>3$ years, $\mathrm{p}<0.0001)$. LCI was elevated in those with respiratory disease compared with healthy controls.

MBW is feasible in a clinic setting in unsedated pre-schoolers, particularly in those $>3$ years old, and LCI is raised in those with respiratory disease. Use of LCI0.5 did not increase success rate in pre-schoolers.

@ERSpublications

Lung clearance index measurement is feasible in children older than 3 years in the clinic http://ow.ly/qcIt300Q6oO

This article has supplementary material available from erj.ersjournals.com

Received: Feb 192016 | Accepted after revision: May 242016 | First published online: July 072016

Support statement: This study was supported by Asthma UK grant AUK-IG-2014-287. A. Bush is an NIHR Senior Investigator and S. Seglani is an NIHR Career Development Fellow (grant CDF-2014-07 019). The project was supported by the NIHR Respiratory Disease Biomedical Research Unit at The Royal Brompton Hospital Foundation Trust and Imperial College London. Funding information for this article has been deposited with the Open Funder Registry.

Conflict of interest: Disclosures can be found alongside this article at erj.ersjournals.com

Copyright $\odot$ ERS 2016 


\section{Introduction}

Pulmonary function tests play an important role in the diagnosis and management of airway diseases. However, their use in pre-school children remains restricted to research and specialised centres. Spirometry is the most commonly used test to assess lung function both in research and in clinical practice, but it requires significant patient co-operation and co-ordination, and is challenging for pre-school children, especially those $<4$ years of age $[1,2]$, and therefore is not routinely used clinically in this age group. The multiple-breath inert gas washout (MBW) test offers an alternative method of obtaining data on paediatric lung function, and is particularly attractive for young children because it requires minimal coordination and only passive co-operation. MBW allows calculation of the lung clearance index (LCI), which is defined as the number of lung turnovers required to washout an inhaled inert gas to $1 / 40$ th of its initial concentration, which in this study we have termed the standard LCI (LCISTD) [3]. The set end-point relates to the sensitivity of the formerly available gas analysers rather than relating to any physiological factor [4]. The best test end-point has yet to be established and various different ones have been suggested. In particular, LCI 0.5 , in which the end point occurs at $1 / 20$ th of the gas concentration rather than $1 / 40$ th [3], requires a shorter test time without loss of information when compared to LCISTD.

LCI is raised in the presence of inflammation, infection, remodelling or other pathology that may lead to ventilation inhomogeneity [5-7] and, to date, has only been extensively utilised within a research setting in pre-school children. This is in part because the low tidal volumes and higher respiratory rates pose a significant challenge to gas analysers. The difficulties of obtaining passive cooperation, combined with long washout times if MBW is used in those with airflow obstruction, in particular, sets logistic challenges for routine use in the clinic. In cystic fibrosis, it has been shown to correlate with high-resolution computed tomography scan results $[8,9]$, to be more frequently abnormal than other infant lung function testing parameters [10] and to predict future deterioration in lung function [11], and it is known to be elevated in children with pre-school multitrigger wheeze [12]. However, success rates are unknown within a clinical setting where test duration may be an important consideration because of limited patient cooperation and time pressures within clinic. We hypothesised that LCI0.5 would significantly increase test feasibility within a clinical setting, in comparison to LCIsTD, in pre-school children (aged 2-5 years). We aimed to determine the feasibility of measuring LCI in pre-school children with a range of airway diseases in a clinic setting.

\section{Methods}

This prospective feasibility study was conducted at the Royal Brompton Hospital, London, UK, from January 2015 to December 2015. All parents and carers gave written, informed consent for their child to participate. Approval was obtained from NRES Committee South East Coast - Brighton \& Sussex, REC number 10/H1101/69.

\section{Subjects}

Children with recurrent wheeze, cystic fibrosis, primary cilia dyskinesia (PCD), recurrent cough and respiratory infections were recruited from the outpatient clinic. Inclusion criteria were age between 2 and 6 years with respiratory disorders diagnosed using conventional criteria [13-15]. Wheeze was doctor diagnosed (by tertiary paediatric respiratory physician) or parent reported, but confirmed using a video questionnaire [16]. A healthy, age-matched control group was recruited from children of colleagues and siblings of patients attending the clinic. Exclusion criteria were preterm ( $<35$ weeks gestation) or weight $<10 \mathrm{~kg}$. A subgroup of patients that were agreeable were asked to return for a follow-up visit to assess if success or failure was consistent on a second occasion.

\section{MBW technique}

The primary objective was to achieve three MBW measures as previously described [17, 18] using a modified photoacoustic gas analyser (Innocor; Innovision, Glamsbjerg, Denmark) [19] during a routine outpatient visit. All measurements were undertaken by one member of staff. Full details are included in the online supplementary material. Children sat upright in a chair (those unwilling to sit alone sat held upright on their parents' laps) with nose clips attached and breathed through a mouthpiece (Medisize, Hillegom, Netherlands). They were encouraged to breathe gently through the mouthpiece whilst watching a DVD playing.

$0.2 \%$ sulfur hexafluoride was used as a tracer gas for the wash-in/out phases. LCI was calculated by dividing the cumulative expired volume by the functional residual capacity (FRC). Any result that had an FRC value $>10 \%$ different from the other two measures was discarded. The end-point was LCISTD, at which the tracer gas reached 1/40th of its original concentration. However, if a child was unable to reach that point, LCI0.5 was used, in which the gas reached 1/20th of its starting value. Any measurement that did not reach LCI0.5 was regarded as a failure. Reasons for failure were noted. A test was judged successful if at least two repeatable traces were obtained. 


\section{Statistical analysis}

Sample size was opportunistic. Demographic data such as height, weight and age are reported as median and range. Group differences in continuous data were analysed using the Mann-Whitney U-test or Kruskal-Wallis one-way ANOVA followed by a Bonferroni correction for more than two groups. $\mathrm{p}<0.05$ was accepted as statistically significant. Chi-squared was used to compare success rates between groups. The Wilcoxon signed rank test was used to assess stability between visits. 95\% confidence limits of the mean differences were used to assess stability of longitudinal data. Data analyses were performed using Graphpad Prism version 6 (Graphpad Software, Inc., San Diego, CA, USA).

\section{Results}

Feasibility of $\mathrm{LCl}$ in preschool children

116 children (median age 4.1 years, range 2.1-5.9 years) were included (table 1). 39 had recurrent wheeze (median age 3.9 years, range 2.1-5.9 years), seven had PCD (median age 5.5 years, range 3.7-5.8 years), 16 had cystic fibrosis (median age 4.9 years, range 2.1-5.9 years), 26 other respiratory conditions (median age 3.8 years, range 2.2-5.6 years) (see supplementary material for details of diagnoses) and 28 healthy controls (median age 4.1 years, range 3.2-5.8 years). 83 (73\%) out of 116 children successfully completed LCISTD measurement. LCI success rates at different ages are shown in figure 1 . Those $<3$ years of age had a success rate of $14 \%$ and those $>3$ years, a success rate of $80 \%(\mathrm{p}<0.0001)$.

On further analysis, no child $<2.5$ years of age completed the test. Between $30 \%$ and $40 \%$ of those aged between 2.5 and 3.5 years completed the test. $85 \%$ of those $>3.5$ years completed the test. Three additional children completed LCI0.5 measurement having been unable to complete a LCISTD measurement. Two of these children were $<3.5$ years old.

Success rate was $70 \%$ for boys and $68 \%$ for girls. 28 (71\%) out of 39 wheezers, 12 (75\%) out of 16 cystic fibrosis patients, six (85\%) out of seven PCD, 15 (57\%) out of 26 children with other respiratory conditions and 20 (71\%) out of 28 healthy controls completed LCIsTD measurement (table 2).

Among all children that completed both LCIsTD and LCI0.5 measurement, the mean time to complete a single washout (to calculate LCISTD) was $67 \mathrm{~s}$ and the time to complete a single washout to $1 / 20$ th of the starting concentration (to calculate LCI0.5) was $50 \mathrm{~s}$, a reduction in test time of $17 \mathrm{~s}$ ( $25 \%$ of test duration), as washin time remains the same in both the LCIsTD and LCI0.5 tests.

\section{Reasons for failed tests}

The reasons for failure to complete LCI testing are summarised in figure 2. The most common was noncooperation/refusal to perform the test (29\%), followed by inability to tolerate the noseclips (19\%), lack of understanding (25\%), inability to maintain a proper seal around the mouth piece (15\%) and being afraid to attempt to the test (12\%). No patients were rejected as all their MBWs were outside the acceptability criteria.

\section{$\mathrm{LCl}$ results in children with respiratory conditions and healthy controls}

Results of LCISTD and LCI0.5 are shown in table 2. Upper limit of normal calculated from these healthy controls was 7.7; there were abnormal results in all disease groups. Healthy controls had a significantly lower LCI compared to children with respiratory diseases other than recurrent wheeze (figure $3 \mathrm{a}$ ). There was no difference in LCI between controls and all wheezers. However, when children with wheeze were divided into

\section{TABLE 1 Demographics}

\begin{tabular}{|c|c|c|c|c|c|}
\hline Children & 28 & 39 & 16 & 7 & 26 \\
\hline Age years median (range) & $4.1(2.11-5.77)$ & $3.9(2.07-5.89)$ & $4.9(2.11-5.95)$ & $5.5(3.66-5.83)$ & $3.8(2.17-5.63)$ \\
\hline Height $\mathrm{cm}$ mean (range) & $102.1(87-115)$ & $103.3(83-121)$ & $104.1(86.7-118.5)$ & $107.7(97.5-113.0)$ & $101.5(88.0-116.5)$ \\
\hline Height centile median (range) & $50(25-98)$ & $50(2-98)$ & 50 (9-98) & $50(25-75)$ & $25(2-99.6)$ \\
\hline Weight kg mean (range) & $21.34(11.40-23.90)$ & $17.40(10.10-23.10)$ & $17.86(13.10-28.00)$ & $17.02(14.80-19.10)$ & $16.10(12.20-22.30)$ \\
\hline BMI centile median (range) & $50(9-99.6)$ & $75(0.4-99.6)$ & $75(9-99.6)$ & $62.5(25-98)$ & $62.5(25-91)$ \\
\hline
\end{tabular}

Healthy control children had a higher body mass index (BMI) than those with respiratory disease. More wheezers were male than female. CF: cystic fibrosis; PCD: primary ciliary dyskinesia. 


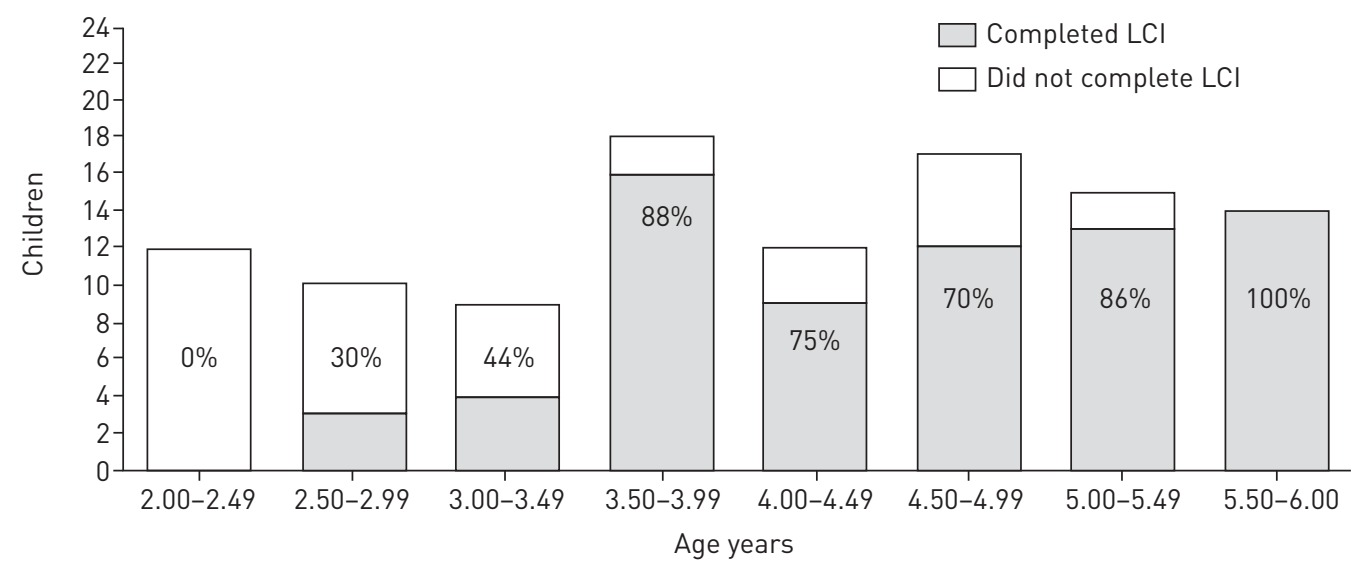

FIGURE 1 Lung clearance index (LCI) success rates according to age. Number of children that completed the standard $\mathrm{LCl}$ measurement in each age group is shown.

those with episodic viral wheeze and those with multitrigger wheeze [20], the multitrigger wheezers had a significantly higher LCI than the episodic viral wheezers (figure $3 \mathrm{~b}$ ) and healthy controls.

Repeat LCl measurements in pre-school children in a clinic setting

19 children underwent MBW on a second visit. The median duration between the first and second visit was 84 days (range 24-210 days). Six (21\%) out of 19 did not complete LCI0.5 or LCIsTD testing on either visit. Two out of 19 completed LCI on the second having been unsuccessful on the first visit. $11(58 \%)$ out of 19 patients successfully completed LCIsTD on both visits (figure 4). Seven patients were clinically stable with no changes to management between visits, while four had changes made to treatment during this period (figure 4). Overall, there was no significant change in LCI between the first and second visit for the whole group (Wilcoxon signed rank test, $\mathrm{p}=0.83$ ) and the $95 \%$ confidence limits of differences between measurements in the seven stable patients were $0.25 \pm 0.32$ of an LCI unit. The coefficient of variation for intervisit repeatability was $3.9 \%$.

\section{Discussion}

We have shown that LCI is feasible in a clinic setting in pre-school children aged between 3.5 and 6 years of age (75-100\% success). However, the success rate in those between 3-3.5 years of age was considerably less (44\%), falling to $30 \%$ for children aged $2.5-3$ years and $0 \%$ in those $<2.5$ years old. Interestingly, and contrary to our hypothesis, LCI0.5 as a test end-point offered no significant increase in success rate compared to LCISTD, but LCI0.5 may increase feasibility rates in children $<3$ years old. There was an overall reduction in washout test time of $17 \mathrm{~s}$ when using the LCI0.5 as the end-point. To our knowledge, this is the first study to investigate feasibility of MBW in pre-school children in $<30 \mathrm{~min}$ and with one member of staff present, simulating a situation that may be viable in a busy outpatient clinic. Success rates overall for those $>3$ years of age were good and these can be used to inform estimated sample sizes in future studies.

The main limitation of the study was the MBW methodology. The tracer gas used was sulfur hexafluoride, which is a greenhouse gas, and future use may be limited by environmental requirements. As a result, nitrogen analysers are increasingly being used. Furthermore, results obtained from nitrogen washout cannot be directly applied to those obtained with sulfur hexafluoride [5]. However, at the time of starting this study, there was no widely accepted pre-school protocol for a nitrogen washout with the commercially

TABLE 2 Lung clearance index (LCI) results and success rates

\begin{tabular}{|c|c|c|c|c|c|c|c|}
\hline & Episodic wheeze & MT wheeze & PCD & CF & Other & Healthy & Total \\
\hline LCI median (range) & $6.05(5.62-8.81)$ & $6.70(6.06-10.84)$ & $7.39(6.39-8.67)$ & $7.09(6.25-13.90)$ & $6.34(5.99-12.00)$ & $6.32(5.39-7.64)$ & $6.43(5.39-13.90)$ \\
\hline LClo.5 median (range) & $4.51(4.19-5.63)$ & $4.64(4.15-6.68)$ & $5.33(4.34-6.02)$ & $5.05(3.22-7.87)$ & $4.73(4.18-7.54)$ & $4.61(3.86-5.71)$ & $4.72(3.22-7.87)$ \\
\hline
\end{tabular}

There were abnormal results in all disease groups. The highest success rates were in primary ciliary dyskinesia (PCD) patients and the lowest in those with other respiratory conditions (not wheeze, cystic fibrosis (CF) or PCD). MT: multitrigger; LClo.5: shortened LCI. 


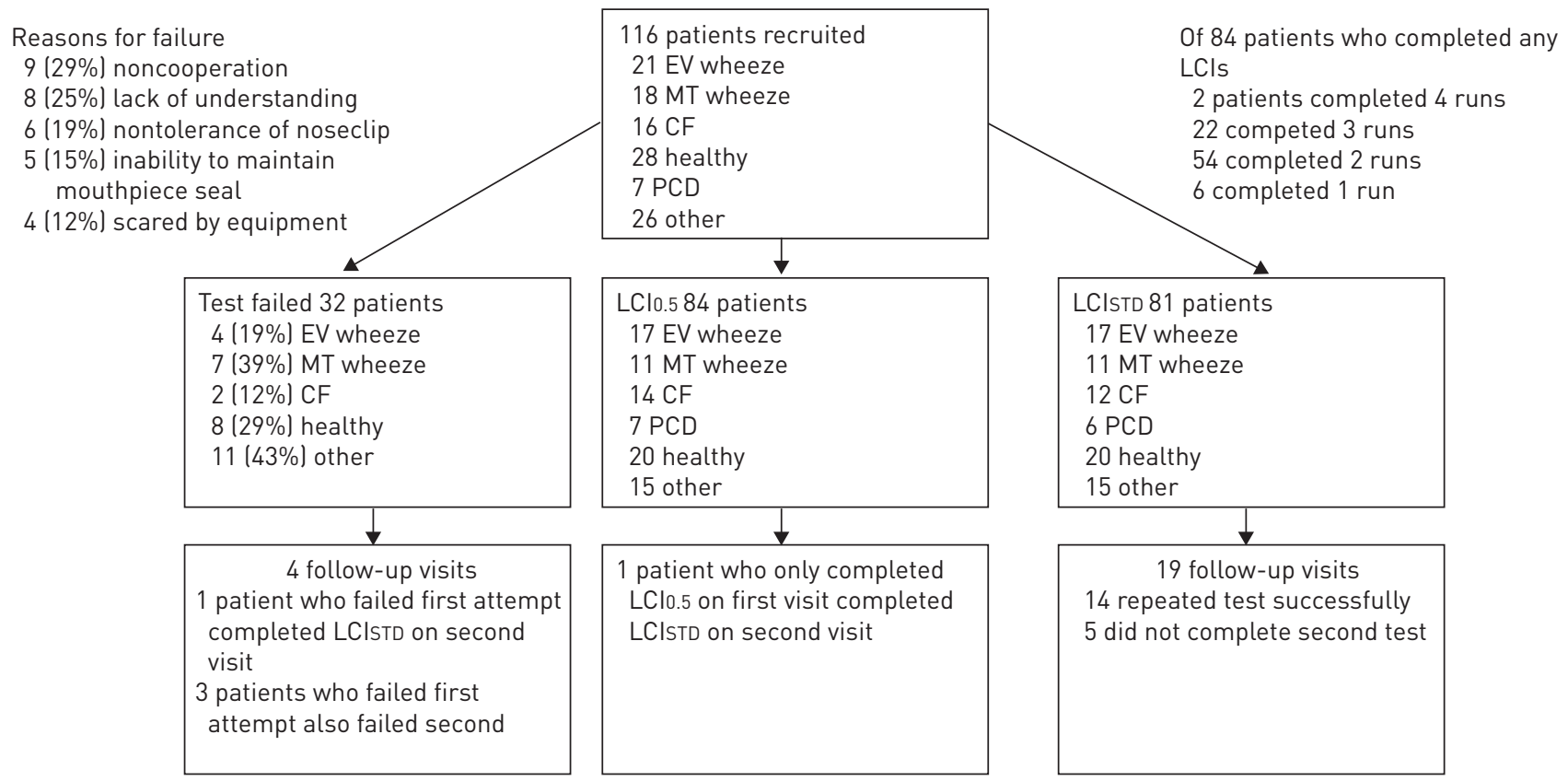

FIGURE 2 Reasons for failure to complete lung clearance index (LCI) testing. EV: episodic viral; MT: multitrigger; CF: cystic fibrosis; PCD: primary ciliary dyskinesia; LCISTD: standard LCl; LClo.5: shortened LCl.

available equipment. In addition, although the photoacoustic gas analyser (Innocor) used met the American Thoracic Society/European Respiratory Society recommendations for children $>10 \mathrm{~kg}$ in weight, this methodology is a custom-made configuration. However, the photoacoustic system is available to measure LCI using a closed-circuit method, which does not have limitations on gas availability, and is currently undergoing validation in pre-schoolers [21]. Overall, it is likely that, since it was child factors that limited success rates, these will be equally applicable to other equipment.

In agreement with published data, we found LCI was raised in children with cystic fibrosis, PCD and multitrigger wheeze, compared to healthy controls, and LCI in multitrigger wheezers was higher than in episodic viral wheeze $[6,18]$. This is important as it shows LCI a clinical setting provided reliable data that reflects previous findings from studies in which the test had been undertaken in a research setting. Moreover, the data suggest LCI may be a sensitive monitoring tool for the management of pre-school wheeze. It has been shown previously that LCI is increased in younger children compared to those aged 6 years and over [22]; as a result, reference equations for calculating $\mathrm{z}$-scored LCI were generated. However, the reference equations were based on mass spectrometry data using a dual-gas washout and using sedation in the youngest subjects. This was felt to be significantly different from our methodology, so we could not assume these equations could be applied. We therefore included contemporaneous healthy controls to make comparisons with our disease patients.

Overall, the success rates in children younger than 3 years were poor, even LCI0.5 was not feasible in this age group, suggesting that unsedated MBW in these patients may not be achievable in the clinical setting.
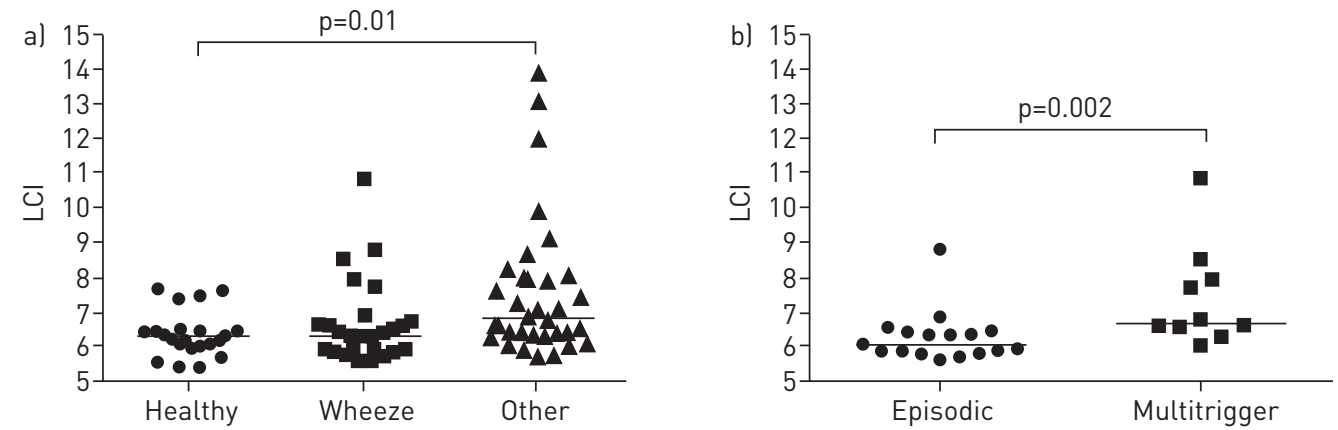

FIGURE 3 a) Lung clearance index (LCI) in healthy controls, wheezers and children with other respiratory disease (including cystic fibrosis and primary ciliary dyskinesia). b) Significantly higher LCl in multitrigger wheezers than episodic wheezers. 


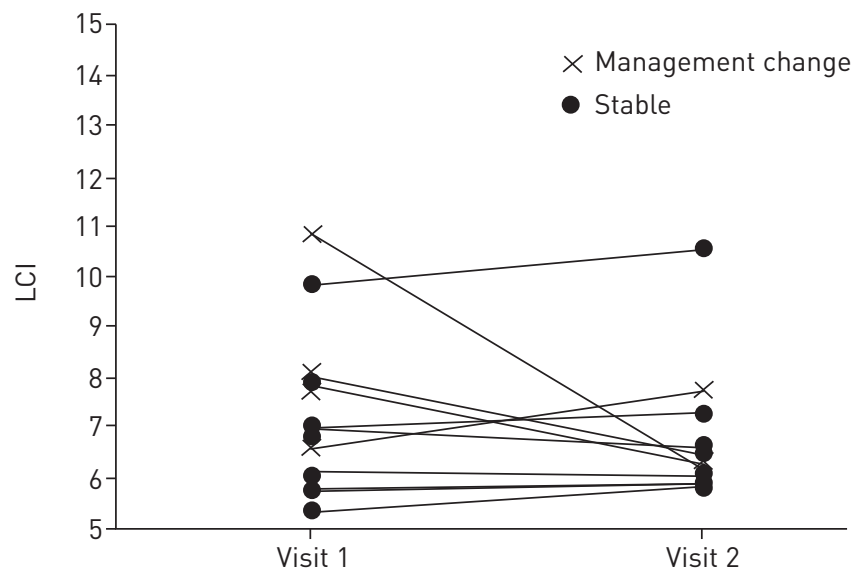

FIGURE 4 Repeatability of lung clearance index (LCI) in a clinic setting. 11 pre-school children had multiple-breath washout measured at two successive clinic appointments. Four out of 11 had management changes following their first visit; seven out of 11 remained stable. There was no significant difference between visits (Wilcoxon signed rank test, $\mathrm{p}=0.83$ ).

However, after age 3 years, results improved and most children completed a technically acceptable MBW that, coupled with the increased LCI seen in some conditions, suggests LCI may be a feasible monitoring tool. Using LCI0.5 did not increase the feasibility compared to LCIsTD. Only three of the 86 children who performed the test for long enough to reach the 1/20th tracer gas concentration cut-off would not continue to the 1/40th standard cut-off. This is an unexpected finding in this group as it was assumed that tolerance of the test would be relatively brief and any time saved would improve success rates. Previous work assessing the usefulness of LCI0.5 was undertaken in school-aged children $[1,3]$; however, pre-school children have a shorter washout period because of a raised respiratory rate and more efficient lung emptying, so the benefit of LCI0.5 may only be relevant in older children and adults.

Previous data on the success of LCI in pre-school children in the research setting have shown an overall success rate of $79 \%$ [7], compared to $73 \%$ in our clinical setting. Success rates in all children $>3.5$ years of age were also extremely good in the research setting (78-87\%) compared to ours in the clinical setting (75-100\%). Even in a research setting, success of LCI measurement in children $<3$ years of age was only $40 \%$. In a clinical setting, this reduced to $30 \%$ in $2.5-3$-year-olds and $0 \%$ in those $<2.5$ years of age.

The coefficient of variation between visits for those seven children who had no management changes was $3.9 \%$. This is comparable to the rate in one previous report (4.2\%) [23] and lower than that seen in control groups in studies that included interventions in patients with cystic fibrosis (9.2\%) [24]; however, the latter group had a considerably higher LCI than our patients.

Surprisingly, intolerance of the noseclip seemed to be a major hurdle in achieving a successful measurement, although this has also been recently reported as a significant source of discomfort in adults [21]. Use of facemasks could reduce this problem and these have been used in other studies, albeit with sedation for the youngest subjects $[7,22,25]$. However, masks also introduce additional dead space, which further reduces the pool of equipment that is suitable for pre-school use and may lead to poor measurements. In addition, use of a facemask often requires two operators (as the mask must be held in place tightly throughout to prevent any leaks), which reduces its application in a busy outpatient clinic. On the basis of the current data, an LCIsTD rather than LCI0.5 should always be attempted in pre-school children. There are several strategies that could be applied to try further to increase the success rate. The first would be to address the issue of noseclips, which proved a common reason for failure. We used standard lung function testing clips manufactured for older children and adults; it may be that a specialist design or possibly commercially available clips designed for comfort when swimming may be more appropriate for preschool children. Although there are drawbacks to using facemasks, it may be that a full feasibility assessment in a clinical situation would be beneficial.

All children who completed the first visit LCI test were able to complete the second visit LCI. This is encouraging, as it suggests that long-term monitoring or multivisit trials will be possible in this age group. Although this group was small, LCI was stable in those with stable disease.

In conclusion, LCI is feasible in an outpatient setting for pre-schoolers aged 3 years and over, although rarely successful in children younger than this. Encouragingly, the results are comparable to those obtained in a research setting. 


\section{Acknowledgements}

We thank all the parents and children who participated in the study, and many thanks to the Respiratory Biomedical Research Unit at the Royal Brompton and Harefield NHS Foundation Trust, London, UK, for their co-operation. We also wish to thank Per Gustafson (University of Gothenburg, Gothenburg, Sweden) for allowing us to use his Washout software programme, Kenneth Macleod (Royal Hospital for Sick Children, Edinburgh, UK) for his help in modifying the Innocor and Fatima Ahmad (Imperial College, London, UK) for developing the shortened LCI methodology used.

\section{References}

1 Yammine S, Singer F, Abbas C, et al. Multiple-breath washout measurements can be significantly shortened in children. Thorax 2013; 68: 586-587.

2 Kampschmidt JC, Brooks EG, Cherry DC, et al. Feasibility of spirometry testing in preschool children. Pediatr Pulmonol 2016; 51: 258-266.

3 Ahmad F, Irving S, Alton E, et al. Multiple breath washouts in children can be shortened without compromising quality. Eur Respir J 2015; 46: 1814-1816.

Horsley A. Lung clearance index in the assessment of airways disease. Respir Med 2009; 103: 793-799.

5 Robinson P, Latzin P, Verbanck S, et al. Consensus statement for inert gas washout measurement using multipleand single-breath tests. Eur Respir J 2013; 41: 507-522.

6 Sonnappa S, Bastardo CM, Saglani S, et al. Relationship between past airway pathology and current lung function in preschool wheezers. Eur Respir J 2011; 38: 1431-1416.

7 Aurora P, Bush A, Gustafsson P, et al. Multiple-breath washout as a marker of lung disease in preschool children with cystic fibrosis. Am J Respir Crit Care Med 2005; 171: 249-256.

8 Ellemunter H, Fuchs SI, Unsinn KM, et al. Sensitivity of lung clearance index and chest computed tomography in early CF lung disease. Respir Med 2010; 104: 1834-1842.

9 Gustafsson PM, De Jong PA, Tiddens HA, et al. Multiple-breath inert gas washout and spirometry versus structural lung disease in cystic fibrosis. Thorax 2008; 63: 129-134.

10 Aurora P, Gustafsson P, Bush A, et al. Multiple breath inert gas washout as a measure of ventilation distribution in children with cystic fibrosis. Thorax 2004; 59: 1068-1073.

11 Aurora P, Stanojevic S, Wade A, et al. Lung clearance index at 4 years predicts subsequent lung function in children with cystic fibrosis. Am J Respir Crit Care Med 2011; 183: 752-758.

12 Sonnappa S, Bastardo CM, Wade A, et al. Repeatability and bronchodilator reversibility of lung function in young children. Eur Respir J 2013; 42: 116-124.

13 Bush A, Chodhari R, Collins N, et al. Primary ciliary dyskinesia: current state of the art. Arch Dis Child 2007; 92: 1136-1140.

14 Rosenstein BJ, Cutting GR. The diagnosis of cystic fibrosis: a consensus statement. Cystic Fibrosis Foundation Consensus Panel. J Pediatr 1998; 132: 589-595.

15 Farrell PM, Rosenstein BJ, White TB, et al. Guidelines for diagnosis of cystic fibrosis in newborns through older adults: Cystic Fibrosis Foundation consensus report. J Pediatr 2008; 153: S4-S14.

16 Saglani S, McKenzie SA, Bush A, et al. A video questionnaire identifies upper airway abnormalities in preschool children with reported wheeze. Arch Dis Child 2005; 90: 961-964.

17 Horsley AR, Gustafsson PM, Macleod KA, et al. Lung clearance index is a sensitive, repeatable and practical measure of airways disease in adults with cystic fibrosis. Thorax 2008; 63: 135-140.

18 Irving SJ, Ives A, Davies G, et al. Lung clearance index and high-resolution computed tomography scores in primary ciliary dyskinesia. Am J Respir Crit Care Med 2013; 188: 545-549.

19 Horsley A, Macleod K, Gupta R, et al. Enhanced photoacoustic gas analyser response time and impact on accuracy at fast ventilation rates during multiple breath washout. PLoS One 2014; 9: e98487.

20 Brand PL, Baraldi E, Bisgaard H, et al. Definition, assessment and treatment of wheezing disorders in preschool children: an evidence-based approach. Eur Respir J 2008; 32: 1096-1110.

21 Horsley AR, O'Neill K, Downey DG, et al. Closed circuit rebreathing to achieve inert gas wash-in for multiple breath wash-out. ERJ Open Research 2016; 2: 00042-2015.

22 Lum S, Stocks J, Stanojevic S, et al. Age and height dependence of lung clearance index and functional residual capacity. Eur Respir J 2013; 41: 1371-1377.

23 Fuchs SI, Eder J, Ellemunter H, et al. Lung clearance index: normal values, repeatability, and reproducibility in healthy children and adolescents. Pediatr Pulmonol 2009; 44: 1180-1185.

24 Amin R, Subbarao P, Jabar A, et al. Hypertonic saline improves the LCI in paediatric patients with CF with normal lung function. Thorax 2010; 65: 379-383. 